\title{
Kernos
}

Revue internationale et pluridisciplinaire de religion grecque antique

22 | 2009

Varia

\section{Sarah Iles Johnston, Ancient Greek Divination}

\section{Antoine Kopij}

\section{OpenEdition}

\section{Journals}

Édition électronique

URL : http://journals.openedition.org/kernos/1800

DOI : 10.4000/kernos. 1800

ISSN : 2034-7871

\section{Éditeur}

Centre international d'étude de la religion grecque antique

\section{Édition imprimée}

Date de publication : 1 janvier 2009

Pagination : 325-327

ISSN : 0776-3824

\section{Référence électronique}

Antoine Kopij, «Sarah lles Johnston, Ancient Greek Divination », Kernos [En ligne], 22 | 2009, mis en ligne le 15 septembre 2011, consulté le 21 septembre 2020. URL : http://journals.openedition.org/ kernos/1800 ; DOI : https://doi.org/10.4000/kernos.1800

Ce document a été généré automatiquement le 21 septembre 2020.

Kernos 


\title{
Sarah Iles Johnston, Ancient Greek Divination
}

\author{
Antoine Kopij
}

\section{RÉFÉRENCE}

Sarah Iles JOHNSTON, Ancient Greek Divination, Oxford, Wiley-Blackwell, 2008. 1 vol. 15 曆昌 $23 \mathrm{~cm}, 193$ p. (Blackwell Ancient Religions). ISBN : 978-1-4051-1573-5.

1 Les Grecs eux-mêmes ont beaucoup discuté de la nature exacte de la divination. Il est en effet primordial de répondre à cette question pour comprendre la place respective des dieux et des hommes dans l'univers, puisque la divination est fondée sur la conviction que les dieux communiquent avec les hommes. Durant le processus, les dieux peuvent répondre aux questions des hommes qui souhaitent connaître le destin qui leur est réservé et les informer de leurs exigences, auxquelles les hommes devront se plier pour réussir leurs entreprises. Dès lors, il était indispensable pour les Grecs de faire intervenir une référence à la divination dans toute représentation philosophicoreligieuse. L'A. parcourt les multiples déclinaisons de ce dialogue dans le monde grec depuis les récits légendaires d'Homère jusqu'au syncrétisme théologique de Proclus, donnant une image aussi claire que possible du rôle important de cet ensemble de pratiques dans la vie des Grecs. Pour ce faire, elle choisit de privilégier la cohérence synchronique aux dépens des variations diachroniques, faisant intervenir des sources parfois distantes dans le temps et l'espace pour soutenir son point de vue, ce qui pourrait lui être reproché. Mais cette démarche offre davantage d'accessibilité qu'un rappel systématique du contexte des données exploitées. Par ailleurs, les références de travaux antérieurs consacrés à la collection et l'organisation du matériel textuel et archéologique sont mises en évidence au début de l'ouvrage.

2 S.I.J. situe son livre dans le sillage direct du volume collectif «Divination et rationalité ", dirigé par Jean-Pierre Vernant et paru en $1974^{1}$, avec lequel elle partage la volonté de décrire le type particulier de rationalité présent dans la procédure 
divinatoire et comment cette dernière s'articule à la structure sociale de son environnement. Au départ de cette recherche réside la conviction, commune à de nombreux chercheurs contemporains, de la nécessité de reconsidérer les catégories discriminantes qui opposent ce que les rationalistes ${ }^{2}$ de l'après-guerre considéraient comme la " rationalité occidentale ", à une altérité caractérisée essentiellement par sa non-conformité aux normes "rationnelles». Il est apparu clairement que cette tendance à donner une connotation péjorative à ce qui nous parait « irrationnel » chez l'autre est une caractéristique de l'esprit humain. Tentant de réparer ce qui était perçu comme une erreur due à une conception culturellement déterminée de la raison humaine, les chercheurs influencés par les apports des différentes sciences humaines se sont ensuite attachés à comprendre ce qui ressortait le plus comme obscur et incompris, à savoir la magie et les rituels d'initiation, laissant de côté la divination, moins exotique justement parce que son ancrage dans la régulation sociale avait déjà été démontré dans l'influent ouvrage de Dodds. C'est pourquoi l'A. a conçu la rédaction d'un ouvrage de synthèse sur la divination grecque.

3 S.I.J. divise sa discussion en deux parties, la première consacrée aux institutional oracles et la deuxième aux independent diviners, considérant que la division héritée de l'antiquité entre divination "naturelle » ou inspirée et "technique » ou apprise, est artificielle et inadéquate au point de vue heuristique. En effet, l'opposition entre divination " naturelle » et " technique » est souvent difficile à situer dans les exemples fournis par les différentes sources. Il semble que, dans la pratique, les deux sortes de divination se recouvraient à des degrés divers. Le premier chapitre d'introduction est suivi par deux chapitres traitant des oracles de Delphes, Dodone, Claros, Didymes et les autres. Le quatrième et le cinquième chapitre traitant de la figure du mantis, avec un accent dans le cinquième et dernier chapitre sur les rapports entre mantis et magicien à l'époque impériale. Pour décrire et expliquer les institutional oracles, l'A. fait intervenir en premier lieu les récits mythologiques, qui nous renseignent sur les particularités religieuses et culturelles propres au lieu de fondation de l'oracle ainsi que sur le type de concept de communication en usage. Après une description succincte mais utile de ce que pouvaient être les impressions du voyageur antique lors de son passage dans le sanctuaire oraculaire et de l'impact de celui-ci sur la vie politique et religieuse des cités grecques, le propos se centre sur la place centrale de celle ou celui par l'intermédiaire de qui s'exprime le dieu. Les individus qui pratiquent la divination sont le thème de la deuxième partie, où ils sont étudiés en dehors du contexte des sanctuaires oraculaires.

$4 \quad$ S.I.J. regroupe sous l'appellation de manteis l'ensemble des individus qui pratiquaient la divination indépendamment des oracles. Il existait un grand nombre de spécialisations distinctes que quelqu'un pouvait proposer contre rémunération, par exemple l'interprétation des rêves ou la lecture de la farine, ou encore l'interprétation d'oracles du passé. Des traits caractéristiques de la divination indépendante étaient l'adaptabilité aux demandes et la proximité de la clientèle, qui ne pouvait pas recourir aux oracles prestigieux et lointains pour des problèmes plus individuels ou quotidiens. La fonction de mantis est intégrée dans le tissu social grec dès une haute époque, comme l'atteste sa présence dans les poèmes homériques. Le mantis y participe aux combats en tant que héros à part entière et chaque armée en possède au moins un, qui assure la communication entre les mondes humain et divin, et personnifie ce rapport. Il est notable que les récits légendaires qui relatent les péripéties des manteis les intègrent d'une part aux arbres généalogiques des grands cycles hellènes, d'autre part aux récits de fondation des oracles. Il en découle une légitimation réciproque qui montre 
l'interdépendance des oracles institutionnels et des manteis. Le tissu social dans lequel sont intégrées ces pratiques divinatoires aux formes multiples se transforme sous l'empire romain. Cette période est marquée par la baisse de fréquentation progressive des sanctuaires oraculaires et l'émergence de nouvelles croyances, dont une, la plus célèbre, aura un rôle primordial dans le changement qui s'opère dans la relation avec le divin.

5 Le christianisme, dès ses premiers temps, qualifie de magiques les pratiques religieuse du paganisme. Par magique, il faut entendre étrange, néfaste. Les dieux du panthéon et leurs subalternes, les daimones, sont attachés à la figure de Satan, l'ennemi de Dieu. S.I.J. démontre qu'une telle condamnation sans équivoque d'un certain type de dévotion n'est possible que dans une théologie dualiste. Dans un système théologique polythéiste, le pratiquant choisit la divinité à qui il s'adresse en fonction du domaine de compétence de cette divinité et se sert des méthodes définies par l'usage pour s'en approcher. Les méthodes de la divination et de la magie de l'époque impériale nous sont connues notamment grâce aux Papyri Graecae Magicae, et par les textes de la théurgie, dont le nom signifie "œuvre divine", qui était une forme de magie placée sous l'autorité des dieux et pratiquée par des néoplatoniciens comme Porphyre et Jamblique. Ces sources montrent l'inconsistance d'une séparation arbitraire entre la divination, comprise comme une pratique religieuse, et la magie, censée s'opposer à celle-ci. L'époque impériale est marquée par l'augmentation de l'interaction culturelle, qui se traduit en termes religieux par l'adoption de nouveaux dieux et des nouvelles pratiques qui leur sont associées. Dans un contexte de transformation sociale, les points de repères ancestraux de la société gréco-romaine qu'étaient les oracles traditionnels ne suffisent plus. La demande grandissante de la population pour une réponse apaisante aux questions aussi bien pragmatiques que théologiques posées par le bouleversement religieux en cours permet à certains individus d'accroître leur prestige. Ceux-ci ont l'avantage d'être facilement accessibles et de pouvoir adapter leurs réponses à leur « clientèle » sans devoir en référer à une tradition rigide, comme dans un oracle institutionnel.

6 Pour conclure, disons que l'A. a choisi d'écrire une synthèse claire, dans le but manifeste de permettre au grand public et aux étudiants de saisir les tenants et les aboutissants de sa réflexion, qu'elle exprime dans un langage pédagogique et non dénué d'humour. Nous ne disposons pas encore de l'ample analyse qui viendrait remplacer le vieil ouvrage de Bouché-Leclercq, mais l'exercice de la synthèse à jour et bien informée est ici pleinement assumé et réussi.

\section{NOTES}

1. J.-P. VERNANT (éd.à, Divination et rationalité, Paris, 1974.

2. Notamment : E.R. DoDDS, The Greeks and the Irrational, Berkeley, 1951 (nombreuses rééditions): M.P. NILSSON, Geschichte der griechischen Religion, 2 vols, Munich 1941-1955 (1967-1974²). 
AUTEURS

ANTOINE KOPIJ

Université de Liège 